\title{
Understanding key drivers of attitudes toward income inequality in the Asia Pacific region
}

\author{
Vo Hong Duc ${ }^{1 *}$, Nguyen Cong Thang ${ }^{1}$, Pham Ngoc Thach ${ }^{1}$, Vo The Anh ${ }^{1}$, Vu Ngoc Tan ${ }^{1}$ \\ ${ }^{1}$ Ho Chi Minh City Open University, Vietnam \\ *Corresponding author: duc.vhong@ou.edu.vn
}

ARTICLE INFO

DOI: $10.46223 / \mathrm{HCMCOUJS}$. econ.en.9.1.175.2019

Received: August $6^{\text {th }}, 2018$

Revised: September $24^{\text {th }}, 2018$

Accepted: March $4^{\text {th }}, 2019$

Keywords:

Asia-Pacific region, attitude toward income inequality, determinants
Attitude toward income inequality and its drivers have attracted great attention from policymakers around the globe. Nevertheless, it appears that there is a shortage of empirical studies on the issue, at least in the context of the Asia-Pacific region - the World's most dynamic economic region. This study is conducted to determine key drivers of attitude toward income inequality from various demographic factors, including Gender, Age, Political party, Education, Supervision, Family income, and Class. Available data for 19 countries at a different level of economic growth and development in the region are collected from the World Values Survey in 2016. The findings from this empirical study suggest that the role of each demographic factor as a significant explanation of variation in the attitude toward income inequality is different across nations in the study. In addition, a set of demographic factors, significantly contributing to the variation in attitude toward income inequality, varies across selected countries in the study. Among the demographic factors, Supervision and Class tend to be dominant factors in explaining variation in the attitude toward income inequality.

\section{Introduction}

In recent years, income inequality and its consequences have attracted attention from economists, academics and policymakers. In its comprehensive study, the Organisation for Economic Co-operation and Development (OECD) demonstrated that in the long-run, income inequality could matter for economic growth (Cingano, 2014). Particularly, income inequality polarizes between social classes, leading to a reduction in the level of trust and cooperation between members within a society. This consequence, in turn, could reduce productivity and investment which are critical inputs of a national economy. Income inequality is also a starting point for various social issues (Dorling, 2011; Stiglitz, 2012). Income inequality is a signal of a concentration of political decision-making which effectively hinders maintaining human resources at the optimal level (Dabla-Norris, Tsounta, Kochhar, Ricka, \& Suphaphiphat, 2015). Moreover, income inequality seems to be associated with poverty in reality, a high rate of crime 
and violence. In the extreme case, income inequality could lead to political instability (DablaNorris et al., 2015; Medgyesi, 2013).

Without exception, income inequality does exist in every nation. For example, in the United States of America, the wealth of the top 1 percent richest individuals accounts for nearly one-third asset of that country as a whole. From 1980 to 2010, the share held by the 1 percent wealthiest population has witnessed a rise in France, United Kingdom, Sweden and Europe. Seriously, to advanced economies and emerging markets alike, inequality in wealth is more observable than that in income which is measured by the Gini index (Dabla-Norris et al., 2015).

Due to the presence of the detrimental effects of income inequality on the society, its wide range of coverage, a true understanding of the extent of income inequality, and its drivers, and how to handle the issue must necessarily become the central focus, from both practical and academic aspects. As such, comprehensive analysis in relation to the attitude toward income inequality, and its drivers, seem to be an inevitable task. From the best of our knowledge, the work of Dabla-Norris et al. (2015) is considered as a pioneering study which focuses on the emerging markets. No study has been found to be conducted with attention to the Asia Pacific region, a new engine of the world economy in the near future. As such, this study is conducted to fill this gap.

The structure of the paper is organized as follows. Following this introduction, literature review is discussed in Section 2. Data and research methodology are both discussed in Section 3. Section 4 presents empirical results. Section 5 concludes and discusses policy implications.

\section{Literature Review}

Social view on inequality is diverse. In contrast to the view of mitigation of inequality due to its detrimental consequences, there is also a view for an acceptable level of inequality, for example, income inequality. Intuitively, as somebody spends more time on work, it is reasonable to pay more for them.

Even, one is ready to tolerate more income inequality in the case when their positions are likely to be improved (Hirschman \& Rothschild, 1973). For that reason, many policies have been initiated in an effort to narrow down the income gap between the rich and the poor.

Medgyesi (2013) stated that structural position was about the influence of one's social position on the views. Particularly, the higher a person's socioeconomic position is, the more income inequality a person believes to be legitimate. Curtis and Andersen (2015) argued that it was the case as economic resources are extremely unequally distributed, emphasizing that the middle class was as likely as the working class to support a reduction in inequality. This conclusion is also consistent with the work of Mau (1997), which demonstrated that for the people in Sweden and Great Britain, who considered themselves as in the bottom of their community, they tended to be in favor of income equality. In early searches on social opinion (Gijsberts, 2002; Noll, 1998), most of them ended up with a finding that people did all share 
egalitarian views rather than income disparity, especially who lived in a nation whose economy was heavily regulated by the government.

Moreover, prior studies revealed that age also constituted an attitude toward income inequality. Austen (2002) found a positive relationship between age and the legitimate ratio of high- to low-status pay. Kelley and Evans (1993), in their interesting note, showed that the older tended to advocate pay differences as compared to the younger by 30 percent. Their conclusion is also consistent with the work of Gijsberts (2002), in fact, the author stated that the older were likely to favor 20 percent more inequality on income than the younger.

Among demographic factors, gender plays a significant impact on attitude toward inequality. The rationale behind is that views on economic inequality between men and women are quite different, due to discriminations and socialization processes (Cyba, 2000; Frerichs, 1997). Austen (2002) argued that in both 1987 and 1992 in Australia, West Germany, and the USA males were in favor of higher legitimate ratio of high- to low-status pay than females. Also, the author emphasized that the magnitude was more observable in the second period (in 1992). At the same time, Gijsberts (2002) confirmed these findings under the context of West Germany, Hungary and Poland.

Another source of variation in attitude toward income inequality is level of education. Intuitively, if one spends more schooling year, they tend to require a higher wage to compensate for educational investment. As a consequence, that legitimate offer could potentially lead to a widening income gap. And that, in its turn, influences the opinion on income inequality. The importance of the educational factor has been investigated (Austen, 2002; Gijsberts, 2002). They all posited that income inequality was in favor of more educated interviewees than fewer ones. In the work of Gijsberts (2002), it was found that the legitimate income inequality was increased by 3 percent as each additional year of schooling, as being the case of Great Britain and the USA. Similarly, 0.6 percent increase in the legitimate ratio of high-to low-status pay was related to each additional year of schooling in Australia (Austen, 2002).

In addition, among all previous studies on the topic, it is widely accepted that political party played an important role in determining social attitude (Austen, 2002; Kim, Huh, Choi, \& Lee, 2018). Especially, from the work of Austen (2002), a significant difference in attitude toward income was observable among people who attended or did not attend political parties. Similar to age and education, the author stated that being a political member also refers to income disparity.

\section{Data and Methodology}

\subsection{Data}

In order to explain variation in attitude toward income inequality through its drivers, this study employs data offered by the World Values Survey (http://www.worldvaluessurvey.org). Using a set of questions to investigate how human beliefs influence social and political life, the operation of the World Values Survey has been covering by almost 100 countries which contain almost 90 percent of the world's population. Its output has been utilized by various studies, government officials, journalists, and students. The most 
current survey - WV6 - was released in 2016. This wave comprises 60 countries in the world. Among them, 19 countries in the Asia Pacific region are selected due to the availability of data. The included countries are Australia, Chile, Taiwan, China, India, Japan, South Korea, Malaysia, New Zealand, Peru, Russia, Thailand, the US, Colombia, Hong Kong, Mexico, Singapore, the Philippines, and Pakistan.

The assessment of dependent variable - attitude toward income inequality - was accomplished by asking respondents to indicate the extent to which they agree on income inequality. Question is in a form of 10-point Likert-type scale in which 1 means incomes should be made more equal and 10 depicts larger income differences as incentives for different individual effort. For a comprehensive view on level of acceptance in countries in the database, means of attitude toward income inequality are demonstrated in Figure 1. Noticeably, among the nineteenth countries, the mean is lowest for Russia while the highest one was found in Pakistan, at about 3.4 and 7.07 respectively. In relation to independent variables, their details are provided in the Appendix.

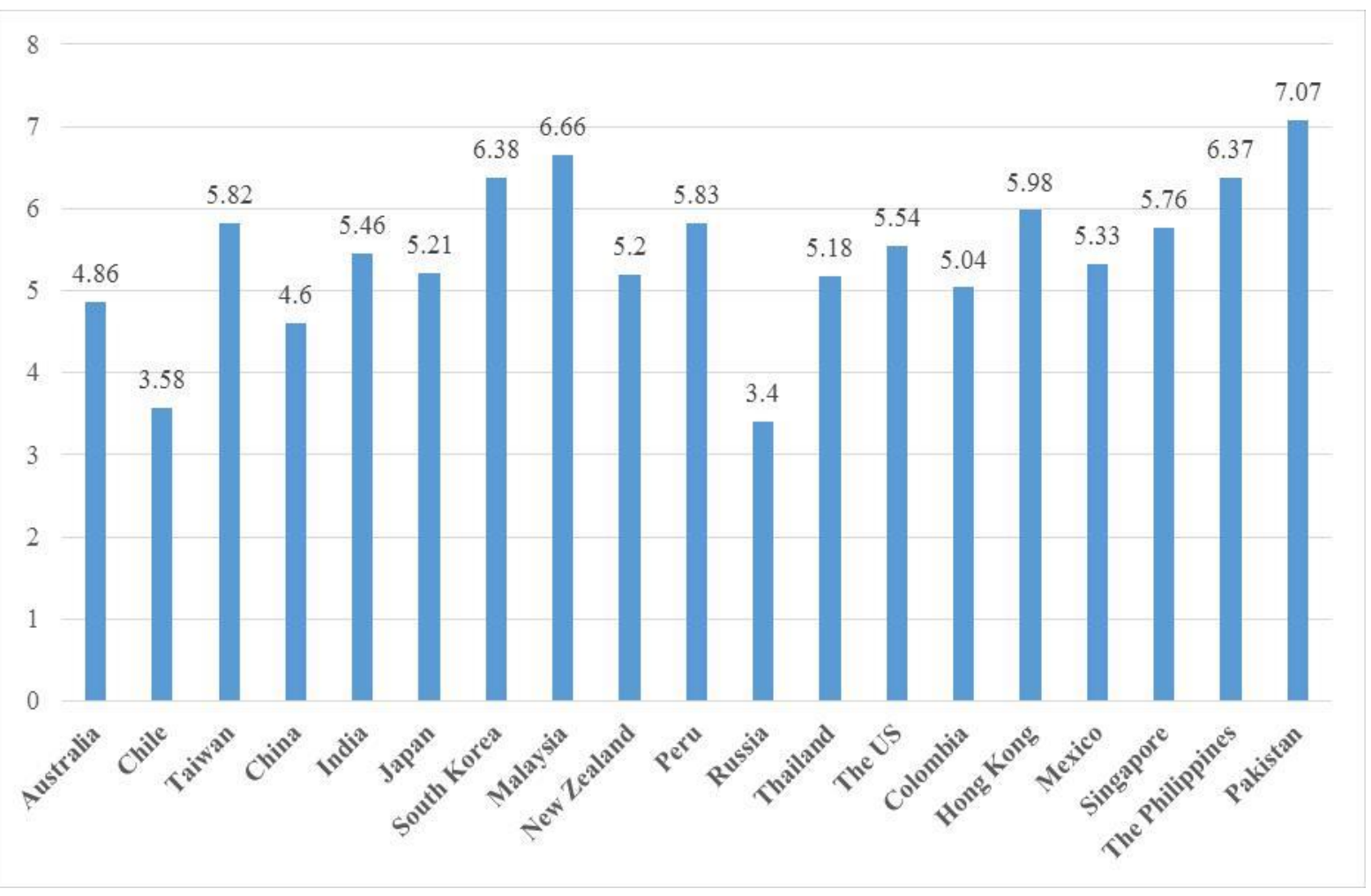

Figure 1. The average score of attitude toward income inequality by countries

Source: Author's calculation.

\subsection{Methodology}

In the scope of this research, the ordered logistics regression is used due to the dependent variable - attitude toward income inequality - is in the form of a qualitative variable which possesses a natural ordering. To clarify how the attitude toward income inequality is explained by its drivers, the following model is taken into account. 


$$
\ln \left(\frac{P_{\text {Attitude }_{i}}}{1-P_{\text {Attitude }_{i}}}\right)=\beta_{0}+\sum \beta_{j} X_{j}+\varepsilon_{i}
$$

Attitude represents for attitude toward income $P_{\text {Attitude }}$ inequality. is the probability of respondents agree on attitude toward income inequality at level i. $\beta_{0}$ is the intercept. $\mathrm{X}_{\mathrm{j}}$ is the set of variables including Gender, Age, Political party, Education, Supervision, Family income, and Class. $\varepsilon_{i}$ is the error term. Moreover, squared value of Age is also included in the model in order to cater for possible curvilinear effects in the relationship between these variables and Attitude toward income inequality (Austen, 2002).

One potential issue in cross-sectional data is that error terms' variances are not equal which may lead to a statistically insignificant coefficient or misleading inferences. As such, White's robust standard error procedure is utilized. In addition, the investigation demonstrated that the foregoing model could encounter the issue of multicollinearity when both variables Class and Family income - are included at the same time. The association between Class and Family income could be the case as a matter of questions utilized in the survey. Therefore, the dependent variable - Attitude toward income inequality - is regressed on Family income and Class separately. The results are reported in Table 2 and Table 3, respectively.

\section{Table 1}

A description of variables

\begin{tabular}{|c|c|}
\hline Variable & Description \\
\hline \multicolumn{2}{|r|}{ Dependent variable } \\
\hline $\begin{array}{l}\text { Attitude toward } \\
\text { income inequality }\end{array}$ & $\begin{array}{l}\text { Measuring income inequality. It is in a form of 10-point Likert-type scale } \\
\text { 1: Income should be made more equal } \\
\text { 10: We need larger income differences as incentive for individual effort }\end{array}$ \\
\hline & Independent variable \\
\hline Gender & $\begin{array}{l}\text { Respondent's gender by observation } \\
\text { 1: Male } \\
0 \text { : Female }\end{array}$ \\
\hline Age & Respondent's age \\
\hline Education & $\begin{array}{l}\text { Measuring the highest education level attended by respondent } \\
\text { 1: No formal education. } \\
\text { 2: Incomplete primary school. } \\
\text { 3: Complete primary school. } \\
\text { 4: Incomplete secondary school: technical/ vocational type. } \\
\text { 5: Complete secondary school: technical/ vocational type. } \\
\text { 6: Incomplete secondary school: university-preparatory type. } \\
\text { 7: Complete secondary school: university-preparatory type. } \\
\text { 8: Some university-level education, without degree. }\end{array}$ \\
\hline
\end{tabular}




\begin{tabular}{|c|c|}
\hline Variable & Description \\
\hline & 9: University - level education, with degree. \\
\hline Family income & $\begin{array}{l}\text { Scale of family income } \\
\text { 1: Lowest group. } \\
\text { 10: Highest group. }\end{array}$ \\
\hline Political party & $\begin{array}{l}\text { Being a member of a political party } \\
0 \text { : Don't belong. } \\
\text { 1: Inactive member. } \\
\text { 2: Active member. }\end{array}$ \\
\hline Supervision & $\begin{array}{l}\text { Supervise or used to supervise other people at word } \\
\text { 1: Yes } \\
0 \text { : No }\end{array}$ \\
\hline Class & $\begin{array}{l}\text { Measuring respondent's social class } \\
\text { 1: Upper class. } \\
\text { 2: Upper middle class. } \\
\text { 3: Lower middle class. } \\
\text { 4: Working class. } \\
\text { 5: Lower class. }\end{array}$ \\
\hline
\end{tabular}

Source: World Values Survey

\section{Empirical Findings}

Table 2 presents empirical findings for each of countries in the sample where Family income was employed instead of Class. First, across 19 countries from the Asia-Pacific region in this study, each demographic factor plays a different role as a significant explanation of variation in the attitude toward income inequality. Second, the component of a set of demographic factors, significantly contributing to the variation in attitude toward income inequality, varies across selected countries in the study.

In relation to the first observation, for example, in Chile, Pakistan, South Korea, and Thailand, Age is a significant factor in explaining variation in the attitude toward income inequality. Moreover, the results also state that there is a difference in the attitude toward income inequality between male and female in Colombia, Malaysia, New Zealand, and the United States. Similarly, the same findings can be reached for Supervision in the context of China, India, Japan, Malaysia, Mexico, Pakistan, Peru, Singapore, and South Korea.

For the odds ratio - the coefficients, to South Korea, it reveals that for one unit increases in Age, the odds of view on "larger income differences as incentives for individual effort" versus the combined of the other views are 0.92 greater, given that all other variables in the model are held constant. Likewise, the odds between the view on "income should be made more equal" and the others also increase 0.92 times for one unit increases in Age. The similar explanations 
are also found in Chile, Pakistan, and Thailand, however, at the magnitude of the odds ratio of 1.04, 0.92 and 1.05, respectively. Indeed, the finding from the older people who favor more income inequality than the younger ones was also found in the works of Gijsberts (2002), and Kelley and Evans (1993).

In terms of Gender, in Malaysia, as compared to female, the odds of view of male on "larger income differences as incentives for individual effort" versus the combined of other views are 0.76 greater. In practice, the studies of Austen (2002), and Gijsberts (2002) also revealed a difference in attitude toward income inequality between male and female. In relation to Supervision, in China, the results suggest that, in the comparison to employees who have not been in charge of supervision, the odds of view on "larger income differences as incentives for individual effort" versus the combined of other views of supervisors are 1.42 larger. Put it differently, it could be seen that the higher a person's socioeconomic position is, the more income inequality a person believes to be legitimate. Prior, the awareness of income difference was influenced by position, which was revealed by Medgyesi (2013), and Mau (1997).

In the context of Australia, the results indicate that the demographic factors - political party, education, family income - significantly explain the change in the attitude toward income inequality whereas a set of supervision, family income is in the case of China. Thus, it is worth noting that a set of demographic factors, which is associated with attitude toward income inequality, varies depending on different countries.

Remarkably, as presented in Table 2, a family income belonging to the range between the fourth group and ninth group, he/she tends to support the income difference rather than income should be made more equal as compared to those whose family income is in the lowest group - the reference category. This finding is also supported in Table 3 where Class is utilized instead of Family income. The results present that as compared to the lower class - the reference category, in 11 countries (e.g., Australia, China, Japan, Malaysia, New Zealand, Russia, Singapore, South Korea, Taiwan, Thailand, and the United States), the participant who describe themselves in the upper-middle-class tend to favor income inequality. 


\section{Table 2}

Ordered logit regression's result by countries. Coefficients are in forms of odds ratio.

\begin{tabular}{|c|c|c|c|c|c|c|c|c|c|c|}
\hline & Australia & Chile & China & Colombia & Hong Kong & India & Japan & Malaysia & Mexico & New Zealand \\
\hline Age & $\begin{array}{c}1.00 \\
(0.021)\end{array}$ & $\begin{array}{c}1.04 * \\
(0.026)\end{array}$ & $\begin{array}{c}1.04 \\
(0.027)\end{array}$ & $\begin{array}{c}1.02 \\
(0.018)\end{array}$ & $\begin{array}{c}1.03 \\
(0.022)\end{array}$ & $\begin{array}{c}1.01 \\
(0.020)\end{array}$ & $\begin{array}{c}1.00 \\
(0.020)\end{array}$ & $\begin{array}{c}0.99 \\
(0.021)\end{array}$ & $\begin{array}{c}1.00 \\
(0.016)\end{array}$ & $\begin{array}{c}0.95 \\
(0.031)\end{array}$ \\
\hline Age squared & $\begin{array}{c}1.00 \\
(0.000)\end{array}$ & $\begin{array}{c}1.00 \\
(0.000)\end{array}$ & $\begin{array}{c}1.00 * \\
(0.000)\end{array}$ & $\begin{array}{c}1.00 \\
(0.000)\end{array}$ & $\begin{array}{c}1.00 \\
(0.000)\end{array}$ & $\begin{array}{c}1.00 \\
(0.000)\end{array}$ & $\begin{array}{c}1.00 \\
(0.000)\end{array}$ & $\begin{array}{c}1.00 \\
(0.000)\end{array}$ & $\begin{array}{c}1.00 \\
(0.000)\end{array}$ & $\begin{array}{c}1.00 \\
(0.000)\end{array}$ \\
\hline Gender & $\begin{array}{c}1.12 \\
(0.137)\end{array}$ & $\begin{array}{c}1.01 \\
(0.140)\end{array}$ & $\begin{array}{c}1.05 \\
(0.112)\end{array}$ & $\begin{array}{l}1.31 * * * \\
(0.133)\end{array}$ & $\begin{array}{c}0.86 \\
(0.103)\end{array}$ & $\begin{array}{c}1.07 \\
(0.125)\end{array}$ & $\begin{array}{c}1.09 \\
(0.108)\end{array}$ & $\begin{array}{c}0.76 * * * \\
(0.079)\end{array}$ & $\begin{array}{c}0.99 \\
(0.092)\end{array}$ & $\begin{array}{c}1.30^{*} \\
(0.198)\end{array}$ \\
\hline Supervision & $\begin{array}{c}0.96 \\
(0.121)\end{array}$ & $\begin{array}{c}0.81 \\
(0.138)\end{array}$ & $\begin{array}{l}1.42 * * * \\
(0.193)\end{array}$ & $\begin{array}{c}1.11 \\
(0.123)\end{array}$ & $\begin{array}{c}0.90 \\
(0.119)\end{array}$ & $\begin{array}{l}0.68 * * * \\
(0.078)\end{array}$ & $\begin{array}{l}1.46 * * * \\
(0.141)\end{array}$ & $\begin{array}{l}1.44 * * * \\
(0.164)\end{array}$ & $\begin{array}{c}0.84^{*} \\
(0.079)\end{array}$ & $\begin{array}{c}1.19 \\
(0.198)\end{array}$ \\
\hline \multicolumn{11}{|l|}{ Political Party } \\
\hline $\begin{array}{l}\text { Inactive } \\
\text { member }\end{array}$ & $\begin{array}{l}0.61 * * * \\
(0.107)\end{array}$ & $\begin{array}{c}0.97 \\
(0.180)\end{array}$ & $\begin{array}{c}1.21 \\
(0.268)\end{array}$ & $\begin{array}{c}1.00 \\
(0.190)\end{array}$ & $\begin{array}{c}1.02 \\
(0.182)\end{array}$ & $\begin{array}{c}1.09 \\
(0.130)\end{array}$ & $\begin{array}{c}0.92 \\
(0.195)\end{array}$ & $\begin{array}{c}0.97 \\
(0.138)\end{array}$ & $\begin{array}{l}1.36 * * \\
(0.194)\end{array}$ & $\begin{array}{l}0.62 * * \\
(0.149)\end{array}$ \\
\hline Active member & $\begin{array}{c}0.65 \\
(0.318)\end{array}$ & $\begin{array}{l}0.37 * * \\
(0.169)\end{array}$ & $\begin{array}{c}0.78 \\
(0.280)\end{array}$ & $\begin{array}{c}1.31 \\
(0.409)\end{array}$ & $\begin{array}{c}0.93 \\
(0.300)\end{array}$ & $\begin{array}{c}1.17 \\
(0.173)\end{array}$ & $\begin{array}{c}0.58 \\
(0.258)\end{array}$ & $\begin{array}{c}1.16 \\
(0.341)\end{array}$ & $\begin{array}{c}1.30 \\
(0.240)\end{array}$ & $\begin{array}{c}0.56 \\
(0.254)\end{array}$ \\
\hline \multicolumn{11}{|l|}{ Education } \\
\hline $1^{\text {st }}$ level & $\begin{array}{c}2.79 \\
(1.893)\end{array}$ & $\begin{array}{c}2.02 * \\
(0.848)\end{array}$ & - & $\begin{array}{c}1.34 \\
(0.279)\end{array}$ & $\begin{array}{c}0.82 \\
(0.415)\end{array}$ & $\begin{array}{c}1.13 \\
(0.221)\end{array}$ & - & $\begin{array}{c}0.94 \\
(0.306)\end{array}$ & $\begin{array}{c}0.99 \\
(0.222)\end{array}$ & - \\
\hline $2^{\text {nd }}$ level & $\begin{array}{c}2.16 \\
(1.030)\end{array}$ & $\begin{array}{c}1.37 \\
(0.544)\end{array}$ & - & $\begin{array}{l}1.69 * * \\
(0.425)\end{array}$ & $\begin{array}{c}0.78 \\
(0.397)\end{array}$ & $\begin{array}{c}1.20 \\
(0.251)\end{array}$ & $\begin{array}{c}1.56 \\
(0.790)\end{array}$ & $\begin{array}{c}1.33 \\
(0.440)\end{array}$ & $\begin{array}{c}1.23 \\
(0.356)\end{array}$ & - \\
\hline $3^{\text {rd }}$ level & $\begin{array}{c}2.01 \\
(0.993)\end{array}$ & $\begin{array}{c}1.36 \\
(0.461)\end{array}$ & $\begin{array}{c}0.80 \\
(0.136)\end{array}$ & $\begin{array}{c}1.02 \\
(0.239)\end{array}$ & $\begin{array}{c}0.89 \\
(0.445)\end{array}$ & $\begin{array}{c}0.97 \\
(0.180)\end{array}$ & $\begin{array}{c}1.03 \\
(0.235)\end{array}$ & $\begin{array}{c}1.06 \\
(0.335)\end{array}$ & $\begin{array}{c}0.87 \\
(0.183)\end{array}$ & - \\
\hline $4^{\text {th }}$ level & $\begin{array}{c}1.54 \\
(0.855)\end{array}$ & $\begin{array}{c}0.87 \\
(0.420)\end{array}$ & - & $\begin{array}{c}1.14 \\
(0.243)\end{array}$ & $\begin{array}{c}0.91 \\
(0.565)\end{array}$ & $\begin{array}{c}0.66^{*} \\
(0.151)\end{array}$ & $\begin{array}{c}0.85 \\
(0.243)\end{array}$ & $\begin{array}{c}1.58 \\
(0.678)\end{array}$ & $\begin{array}{c}1.07 \\
(0.257)\end{array}$ & $\begin{array}{c}5.20 \\
(7.955)\end{array}$ \\
\hline $5^{\text {th }}$ level & $\begin{array}{c}2.66 * \\
(1.380)\end{array}$ & $\begin{array}{c}0.96 \\
(0.374)\end{array}$ & $\begin{array}{c}0.90 \\
(0.157)\end{array}$ & $\begin{array}{l}1.50 * * \\
(0.303)\end{array}$ & $\begin{array}{c}0.65 \\
(0.332)\end{array}$ & $\begin{array}{c}1.25 \\
(0.283)\end{array}$ & $\begin{array}{c}1.12 \\
(0.214)\end{array}$ & $\begin{array}{c}1.13 \\
(0.383)\end{array}$ & $\begin{array}{c}1.10 \\
(0.245)\end{array}$ & $\begin{array}{c}3.60 \\
(5.515)\end{array}$ \\
\hline
\end{tabular}




\begin{tabular}{|c|c|c|c|c|c|c|c|c|c|c|}
\hline $6^{\text {th }}$ level & $\begin{array}{l}2.97 * * \\
(1.521)\end{array}$ & $\begin{array}{c}0.67 \\
(0.284)\end{array}$ & - & $\begin{array}{c}1.60 \\
(0.357)\end{array}$ & $\begin{array}{c}0.74 \\
(0.399)\end{array}$ & $\begin{array}{c}0.81 \\
(0.192)\end{array}$ & $\begin{array}{c}1.70 \\
(0.754)\end{array}$ & $\begin{array}{l}2.14 * * \\
(0.754)\end{array}$ & $\begin{array}{c}0.69 \\
(0.191)\end{array}$ & $\begin{array}{c}4.11 \\
(6.357)\end{array}$ \\
\hline $7^{\text {th }}$ level & $\begin{array}{c}2.23 * \\
(1.069)\end{array}$ & $\begin{array}{c}0.95 \\
(0.375)\end{array}$ & $\begin{array}{c}1.06 \\
(0.211)\end{array}$ & $\begin{array}{c}1.39 \\
(0.298)\end{array}$ & $\begin{array}{c}0.82 \\
(0.417)\end{array}$ & $\begin{array}{c}3.88 * * * \\
(1.190)\end{array}$ & $\begin{array}{c}1.37 \\
(0.288)\end{array}$ & $\begin{array}{c}1.49 \\
(0.549)\end{array}$ & $\begin{array}{c}0.88 \\
(0.196)\end{array}$ & $\begin{array}{c}3.94 \\
(6.091)\end{array}$ \\
\hline \multicolumn{11}{|l|}{ Family income } \\
\hline $2^{\text {nd }}$ group & $\begin{array}{c}2.09 * \\
(0.794)\end{array}$ & $\begin{array}{c}2.47 \\
(1.469)\end{array}$ & $\begin{array}{c}1.57 \\
(0.559)\end{array}$ & $\begin{array}{c}0.93 \\
(0.298)\end{array}$ & $\begin{array}{c}1.02 \\
(0.522)\end{array}$ & $\begin{array}{c}1.12 \\
(0.256)\end{array}$ & $\begin{array}{c}1.13 \\
(0.175)\end{array}$ & $\begin{array}{c}1.47 \\
(0.956)\end{array}$ & $\begin{array}{c}0.94 \\
(0.127)\end{array}$ & $\begin{array}{c}1.19 \\
(0.566)\end{array}$ \\
\hline $3^{\text {rd }}$ group & $\begin{array}{c}1.54 \\
(0.526)\end{array}$ & $\begin{array}{c}2.79 * \\
(1.623)\end{array}$ & $\begin{array}{c}1.23 \\
(0.378)\end{array}$ & $\begin{array}{c}1.21 \\
(0.353)\end{array}$ & $\begin{array}{c}1.05 \\
(0.461)\end{array}$ & $\begin{array}{c}1.65^{*} \\
(0.455)\end{array}$ & $\begin{array}{l}1.68 * * * \\
(0.269)\end{array}$ & $\begin{array}{c}0.43 \\
(0.234)\end{array}$ & $\begin{array}{c}1.02 \\
(0.153)\end{array}$ & $\begin{array}{c}0.96 \\
(0.406)\end{array}$ \\
\hline $4^{\text {th }}$ group & $\begin{array}{l}2.01 * * \\
(0.652)\end{array}$ & $\begin{array}{l}4.03 * * \\
(2.308)\end{array}$ & $\begin{array}{c}1.61 \\
(0.484)\end{array}$ & $\begin{array}{c}1.05 \\
(0.296)\end{array}$ & $\begin{array}{c}1.14 \\
(0.501)\end{array}$ & $\begin{array}{c}1.36^{*} \\
(0.329)\end{array}$ & $\begin{array}{l}1.92 * * * \\
(0.337)\end{array}$ & $\begin{array}{c}0.37 * * \\
(0.200)\end{array}$ & $\begin{array}{c}1.04 \\
(0.168)\end{array}$ & $\begin{array}{c}1.03 \\
(0.414)\end{array}$ \\
\hline $5^{\text {th }}$ group & $\begin{array}{l}2.50 * * * \\
(0.797)\end{array}$ & $\begin{array}{l}6.85^{* * * *} \\
(3.929)\end{array}$ & $\begin{array}{c}1.33 \\
(0.396)\end{array}$ & $\begin{array}{c}1.50 \\
(0.402)\end{array}$ & $\begin{array}{c}1.36 \\
(0.595)\end{array}$ & $\begin{array}{l}1.59 * * * \\
(0.395)\end{array}$ & $\begin{array}{c}1.40 * \\
(0.246)\end{array}$ & $\begin{array}{c}0.28 * * * \\
(0.138)\end{array}$ & $\begin{array}{c}0.91 \\
(0.163)\end{array}$ & $\begin{array}{c}1.53 \\
(0.647)\end{array}$ \\
\hline $6^{\text {th }}$ group & $\begin{array}{c}2.64 * * * \\
(0.862)\end{array}$ & $\begin{array}{c}8.00 \\
(4.639)\end{array}$ & $\begin{array}{c}1.43 \\
(0.438)\end{array}$ & $\begin{array}{c}1.15 \\
(0.322)\end{array}$ & $\begin{array}{c}1.24 \\
(0.548)\end{array}$ & $\begin{array}{c}3.14 * * * \\
(0.802)\end{array}$ & $\begin{array}{c}1.64 * * * \\
(0.282)\end{array}$ & $\begin{array}{c}0.34 * * \\
(0.163)\end{array}$ & $\begin{array}{c}0.94 \\
(0.203)\end{array}$ & $\begin{array}{c}1.18 \\
(0.496)\end{array}$ \\
\hline $7^{\text {th }}$ group & $\begin{array}{l}3.57 * * * \\
(1.149)\end{array}$ & $\begin{array}{c}8.10 * * * \\
(4.861)\end{array}$ & $\begin{array}{c}1.48 \\
(0.464)\end{array}$ & $\begin{array}{c}1.41 \\
(0.405)\end{array}$ & $\begin{array}{c}1.71 \\
(0.747)\end{array}$ & $\begin{array}{l}3.58 * * * \\
(0.959)\end{array}$ & $\begin{array}{c}1.93 * * \\
(0.508)\end{array}$ & $\begin{array}{c}0.42 * \\
(0.201)\end{array}$ & $\begin{array}{c}0.94 \\
(0.168)\end{array}$ & $\begin{array}{c}1.64 \\
(0.695)\end{array}$ \\
\hline $8^{\text {th }}$ group & $\begin{array}{c}4.13 * * * \\
(1.445)\end{array}$ & $\begin{array}{c}10.64 * * * \\
(6.705)\end{array}$ & $\begin{array}{c}1.48 \\
(0.503)\end{array}$ & $\begin{array}{c}1.51 \\
(0.477)\end{array}$ & $\begin{array}{c}1.40 \\
(0.695)\end{array}$ & $\begin{array}{c}3.63 * * * \\
(0.973)\end{array}$ & $\begin{array}{c}2.38 * * * \\
(0.514)\end{array}$ & $\begin{array}{c}0.43^{*} \\
(0.210)\end{array}$ & $\begin{array}{c}1.06 \\
(0.227)\end{array}$ & $\begin{array}{c}1.66 \\
(0.694)\end{array}$ \\
\hline $9^{\text {th }}$ group & $\begin{array}{l}4.45^{* * * *} \\
(2.096)\end{array}$ & $\begin{array}{l}7.49 * * * \\
(5.181)\end{array}$ & $\begin{array}{c}2.73 * \\
(1.448)\end{array}$ & $\begin{array}{c}0.60 \\
(0.376)\end{array}$ & $\begin{array}{c}1.96 \\
(1.827)\end{array}$ & $\begin{array}{c}4.59 * * * \\
(1.342)\end{array}$ & $\begin{array}{c}2.51 * * * \\
(0.507)\end{array}$ & $\begin{array}{c}0.40 \\
(0.238)\end{array}$ & $\begin{array}{c}0.65 \\
(0.206)\end{array}$ & $\begin{array}{c}2.12 * \\
(0.906)\end{array}$ \\
\hline Highest group & $\begin{array}{c}13.37 * * * \\
(10.217)\end{array}$ & $\begin{array}{c}36.85 * * * \\
(23.996)\end{array}$ & $\begin{array}{r}27.24 * * * \\
(26.145)\end{array}$ & $\begin{array}{l}3.80 * * \\
(2.555)\end{array}$ & $\begin{array}{c}9.34 \\
(15.894)\end{array}$ & $\begin{array}{c}12.06 * * * \\
(4.815)\end{array}$ & $\begin{array}{c}3.27 * * * \\
(0.671)\end{array}$ & $\begin{array}{c}0.74 \\
(0.612)\end{array}$ & $\begin{array}{l}2.56 * * \\
(1.092)\end{array}$ & $\begin{array}{c}2.95 * * \\
(1.288)\end{array}$ \\
\hline Num of obs & 963 & 782 & 1135 & 1291 & 933 & 1190 & 1718 & 1182 & 1579 & 596 \\
\hline Pseudo R2 & 0.0176 & 0.0244 & 0.0094 & 0.0086 & 0.116 & 0.0361 & 0.0163 & 0.0136 & 0.004 & 0.0182 \\
\hline Prob > chi2 & 0.000 & 0.000 & 0.000 & 0.004 & 0.006 & 0.000 & 0.000 & 0.000 & 0.317 & 0.000 \\
\hline
\end{tabular}


Table 2

Ordered logit regression's result by countries. Coefficients are in forms of odds ratio. (cont'd)

\begin{tabular}{|c|c|c|c|c|c|c|c|c|c|}
\hline & Pakistan & Peru & Russia & Singapore & South Korea & Taiwan & Thailand & The Philippines & United States \\
\hline Age & $\begin{array}{l}0.92 * * \\
(0.037)\end{array}$ & $\begin{array}{c}1.01 \\
(0.018)\end{array}$ & $\begin{array}{c}1.00 \\
(0.015)\end{array}$ & $\begin{array}{c}1.00 \\
(0.014)\end{array}$ & $\begin{array}{c}0.92 * * * \\
(0.022)\end{array}$ & $\begin{array}{c}0.98 \\
(0.023)\end{array}$ & $\begin{array}{c}1.05^{*} \\
(0.031)\end{array}$ & $\begin{array}{c}1.00 \\
(0.020)\end{array}$ & $\begin{array}{c}0.98 \\
(0.012)\end{array}$ \\
\hline Age squared & $\begin{array}{l}1.00 * * \\
(0.000)\end{array}$ & $\begin{array}{c}1.00 \\
(0.000)\end{array}$ & $\begin{array}{c}1.00 * \\
(0.000)\end{array}$ & $\begin{array}{c}1.00 \\
(0.000)\end{array}$ & $\begin{array}{c}1.00 * * * \\
(0.000)\end{array}$ & $\begin{array}{c}1.00 * \\
(0.000)\end{array}$ & $\begin{array}{c}1.00 * \\
(0.000)\end{array}$ & $\begin{array}{c}1.00 \\
(0.000)\end{array}$ & $\begin{array}{c}1.00 \\
(0.000)\end{array}$ \\
\hline Gender & $\begin{array}{c}0.59 \\
(0.422)\end{array}$ & $\begin{array}{c}1.04 \\
(0.118)\end{array}$ & $\begin{array}{c}1.07 \\
(0.090)\end{array}$ & $\begin{array}{c}0.96 \\
(0.082)\end{array}$ & $\begin{array}{c}1.08 \\
(0.124)\end{array}$ & $\begin{array}{c}0.91 \\
(0.118)\end{array}$ & $\begin{array}{c}1.02 \\
(0.111)\end{array}$ & $\begin{array}{c}0.98 \\
(0.112)\end{array}$ & $\begin{array}{c}1.24 * * * \\
(0.098)\end{array}$ \\
\hline Supervision & $\begin{array}{c}2.15 * * * \\
(0.468)\end{array}$ & $\begin{array}{c}1.09 * * \\
(0.153)\end{array}$ & $\begin{array}{c}1.30 \\
(0.137)\end{array}$ & $\begin{array}{c}1.27 * * * \\
(0.116)\end{array}$ & $\begin{array}{c}1.34 * * \\
(0.190)\end{array}$ & $\begin{array}{c}1.10 \\
(0.157)\end{array}$ & $\begin{array}{c}0.96 \\
(0.126)\end{array}$ & $\begin{array}{c}1.19 \\
(0.150)\end{array}$ & $\begin{array}{c}1.14 \\
(0.092)\end{array}$ \\
\hline \multicolumn{10}{|l|}{ Political Party } \\
\hline Inactive member & $\begin{array}{l}2.36 * * \\
(0.924)\end{array}$ & $\begin{array}{c}0.80 \\
(0.214)\end{array}$ & $\begin{array}{c}1.50 \\
(0.431)\end{array}$ & $\begin{array}{c}0.85 \\
(0.122)\end{array}$ & $\begin{array}{c}0.72 \\
(0.178)\end{array}$ & $\begin{array}{c}1.26 \\
(0.207)\end{array}$ & $\begin{array}{c}0.97 \\
(0.202)\end{array}$ & $\begin{array}{c}1.11 \\
(0.201)\end{array}$ & $\begin{array}{c}1.02 \\
(0.088)\end{array}$ \\
\hline Active member & $\begin{array}{c}1.93 \\
(0.830)\end{array}$ & $\begin{array}{c}1.16 \\
(0.533)\end{array}$ & $\begin{array}{c}1.64 \\
(0.902)\end{array}$ & $\begin{array}{c}1.04 \\
(0.255)\end{array}$ & $\begin{array}{c}0.32 \\
(0.237)\end{array}$ & $\begin{array}{c}1.41 \\
(0.532)\end{array}$ & $\begin{array}{c}1.86 * * \\
(0.540)\end{array}$ & $\begin{array}{c}1.21 \\
(0.273)\end{array}$ & $\begin{array}{c}1.04 \\
(0.135)\end{array}$ \\
\hline \multicolumn{10}{|l|}{ Education } \\
\hline $1^{\text {st }}$ level & $\begin{array}{l}2.86 * * \\
(1.219)\end{array}$ & $\begin{array}{c}1.26 \\
(0.376)\end{array}$ & $\begin{array}{c}0.88 \\
(0.595)\end{array}$ & $\begin{array}{c}1.16 \\
(0.271)\end{array}$ & $\begin{array}{c}1.55 \\
(0.833)\end{array}$ & $\begin{array}{c}1.10 \\
(0.638)\end{array}$ & $\begin{array}{c}0.91 \\
(0.237)\end{array}$ & $\begin{array}{c}0.83 \\
(0.197)\end{array}$ & $\begin{array}{c}1.01 \\
(0.684)\end{array}$ \\
\hline $2^{\text {nd }}$ level & $\begin{array}{l}2.36 * * \\
(0.864)\end{array}$ & $\begin{array}{c}1.31 \\
(0.352)\end{array}$ & $\begin{array}{c}1.42 \\
(0.833)\end{array}$ & $\begin{array}{c}1.00 \\
(0.254)\end{array}$ & $\begin{array}{c}1.78 \\
(1.352)\end{array}$ & $\begin{array}{l}0.20 * * \\
(0.137)\end{array}$ & $\begin{array}{c}1.12 \\
(0.484)\end{array}$ & $\begin{array}{c}0.80 \\
(0.235)\end{array}$ & - \\
\hline $3^{\text {rd }}$ level & $\begin{array}{c}1.84 \\
(0.718)\end{array}$ & $\begin{array}{l}1.82 * * \\
(0.448)\end{array}$ & $\begin{array}{c}0.78 \\
(0.427)\end{array}$ & $\begin{array}{c}1.22 \\
(0.249)\end{array}$ & $\begin{array}{c}1.01 \\
(0.490)\end{array}$ & $\begin{array}{c}0.85 \\
(0.486)\end{array}$ & $\begin{array}{c}1.13 \\
(0.338)\end{array}$ & $\begin{array}{c}0.72 \\
(0.174)\end{array}$ & - \\
\hline
\end{tabular}




\begin{tabular}{|c|c|c|c|c|c|c|c|c|c|}
\hline & Pakistan & Peru & Russia & Singapore & South Korea & Taiwan & Thailand & $\begin{array}{l}\text { The } \\
\text { Philippines }\end{array}$ & United States \\
\hline $4^{\text {th }}$ level & $\begin{array}{c}2.25 \\
(1.311)\end{array}$ & $\begin{array}{l}1.90 * * \\
(0.580)\end{array}$ & $\begin{array}{c}0.92 \\
(0.511)\end{array}$ & $\begin{array}{c}1.23 \\
(0.299)\end{array}$ & $\begin{array}{c}1.38 \\
(0.731)\end{array}$ & $\begin{array}{c}0.74 \\
(0.585)\end{array}$ & $\begin{array}{c}0.60 \\
(0.283)\end{array}$ & $\begin{array}{c}1.12 \\
(0.312)\end{array}$ & $\begin{array}{c}0.72 \\
(0.414)\end{array}$ \\
\hline $5^{\text {th }}$ level & $\begin{array}{c}2.19 * \\
(0.994)\end{array}$ & $\begin{array}{c}2.22 * * * \\
(0.603)\end{array}$ & $\begin{array}{c}0.69 \\
(0.377)\end{array}$ & $\begin{array}{c}0.90 \\
(0.202)\end{array}$ & $\begin{array}{c}1.40 \\
(0.644)\end{array}$ & $\begin{array}{c}0.85 \\
(0.480)\end{array}$ & $\begin{array}{c}0.69 \\
(0.230)\end{array}$ & $\begin{array}{c}0.99 \\
(0.234)\end{array}$ & $\begin{array}{c}0.74 \\
(0.414)\end{array}$ \\
\hline $6^{\text {th }}$ level & $\begin{array}{c}1.54 \\
(0.657)\end{array}$ & $\begin{array}{c}2.23 * * * \\
(0.627)\end{array}$ & $\begin{array}{c}1.01 \\
(0.574)\end{array}$ & $\begin{array}{c}0.68 \\
(0.186)\end{array}$ & $\begin{array}{c}1.41 \\
(0.687)\end{array}$ & $\begin{array}{c}1.44 \\
(0.977)\end{array}$ & $\begin{array}{c}0.22 \\
(0.206)\end{array}$ & $\begin{array}{c}0.96 \\
(0.219)\end{array}$ & $\begin{array}{c}0.72 \\
(0.407)\end{array}$ \\
\hline $7^{\text {th }}$ level & $\begin{array}{l}3.28 * * \\
(1.658)\end{array}$ & $\begin{array}{l}1.91 * * \\
(0.517)\end{array}$ & $\begin{array}{c}0.84 \\
(0.462)\end{array}$ & $\begin{array}{c}0.93 \\
(0.205)\end{array}$ & $\begin{array}{c}1.08 \\
(0.509)\end{array}$ & $\begin{array}{c}0.92 \\
(0.520)\end{array}$ & $\begin{array}{l}0.51 * * \\
(0.151)\end{array}$ & $\begin{array}{c}1.50^{*} \\
(0.368)\end{array}$ & $\begin{array}{c}0.56 \\
(0.314)\end{array}$ \\
\hline \multicolumn{10}{|c|}{ Family income } \\
\hline $2^{\text {nd }}$ group & $\begin{array}{c}2.91 \\
(3.364)\end{array}$ & $\begin{array}{c}2.82 * * * \\
(1.091)\end{array}$ & $\begin{array}{c}0.96 \\
(0.233)\end{array}$ & $\begin{array}{c}0.84 \\
(1.057)\end{array}$ & $\begin{array}{c}0.80 \\
(0.334)\end{array}$ & $\begin{array}{c}0.44 \\
(0.282)\end{array}$ & $\begin{array}{c}0.57 * \\
(0.170)\end{array}$ & $\begin{array}{c}0.70 \\
(0.197)\end{array}$ & $\begin{array}{c}1.55 \\
(0.543)\end{array}$ \\
\hline $3^{\text {rd }}$ group & $\begin{array}{c}1.68 \\
(1.907)\end{array}$ & $\begin{array}{c}2.46 * * * \\
(0.821)\end{array}$ & $\begin{array}{c}1.20 \\
(0.246)\end{array}$ & $\begin{array}{c}2.68 \\
(3.327)\end{array}$ & $\begin{array}{c}0.52 * \\
(0.197)\end{array}$ & $\begin{array}{c}0.53 \\
(0.306)\end{array}$ & $\begin{array}{c}1.33 \\
(0.405)\end{array}$ & $\begin{array}{c}0.77 \\
(0.181)\end{array}$ & $\begin{array}{c}1.18 \\
(0.357)\end{array}$ \\
\hline $4^{\text {th }}$ group & $\begin{array}{c}1.66 \\
(1.861)\end{array}$ & $\begin{array}{c}2.83 * * * \\
(0.904)\end{array}$ & $\begin{array}{l}1.53 * * \\
(0.320)\end{array}$ & $\begin{array}{c}2.61 \\
(3.203)\end{array}$ & $\begin{array}{c}0.56^{*} \\
(0.194)\end{array}$ & $\begin{array}{c}0.64 \\
(0.371)\end{array}$ & $\begin{array}{c}1.03 \\
(0.241)\end{array}$ & $\begin{array}{c}0.93 \\
(0.228)\end{array}$ & $\begin{array}{c}1.54 \\
(0.455)\end{array}$ \\
\hline $5^{\text {th }}$ group & $\begin{array}{c}1.37 \\
(1.535)\end{array}$ & $\begin{array}{c}2.79 * * * \\
(0.881)\end{array}$ & $\begin{array}{c}1.82 * * * \\
(0.371)\end{array}$ & $\begin{array}{c}2.70 \\
(3.296)\end{array}$ & $\begin{array}{c}0.54^{*} \\
(0.186)\end{array}$ & $\begin{array}{c}0.68 \\
(0.388)\end{array}$ & $\begin{array}{l}1.56 * * * \\
(0.266)\end{array}$ & $\begin{array}{c}0.75 \\
(0.159)\end{array}$ & $\begin{array}{l}1.82 * * \\
(0.523)\end{array}$ \\
\hline $6^{\text {th }}$ group & $\begin{array}{c}0.84 \\
(0.929)\end{array}$ & $\begin{array}{c}3.00 * * * \\
(0.946)\end{array}$ & $\begin{array}{c}1.78 * * * \\
(0.384)\end{array}$ & $\begin{array}{c}3.71 \\
(4.538)\end{array}$ & $\begin{array}{c}0.74 \\
(0.259)\end{array}$ & $\begin{array}{c}0.94 \\
(0.549)\end{array}$ & $\begin{array}{l}1.59 * * * \\
(0.277)\end{array}$ & $\begin{array}{c}0.70 \\
(0.162)\end{array}$ & $\begin{array}{c}2.41 * * * \\
(0.704)\end{array}$ \\
\hline $7^{\text {th }}$ group & $\begin{array}{c}1.61 \\
(1.789)\end{array}$ & $\begin{array}{c}5.61 * * * \\
(1.866)\end{array}$ & $\begin{array}{c}1.97 * * * \\
(0.496)\end{array}$ & $\begin{array}{c}4.16 \\
(5.080)\end{array}$ & $\begin{array}{c}0.81 \\
(0.287)\end{array}$ & $\begin{array}{c}1.50 \\
(0.882)\end{array}$ & $\begin{array}{l}1.66 * * * \\
(0.283)\end{array}$ & $\begin{array}{c}0.90 \\
(0.226)\end{array}$ & $\begin{array}{l}2.54 * * \\
(0.756)\end{array}$ \\
\hline $8^{\text {th }}$ group & $\begin{array}{c}2.35 \\
(2.599)\end{array}$ & $\begin{array}{c}4.79 * * * \\
(2.271)\end{array}$ & $\begin{array}{c}2.18^{* * * *} \\
(0.655)\end{array}$ & $\begin{array}{c}6.05 \\
(7.444)\end{array}$ & $\begin{array}{c}1.28 \\
(0.519)\end{array}$ & $\begin{array}{c}0.83 \\
(0.535)\end{array}$ & $\begin{array}{l}1.96^{* * *} \\
(0.460)\end{array}$ & $\begin{array}{c}0.78 \\
(0.231)\end{array}$ & $\begin{array}{c}3.50 * * * \\
(1.108)\end{array}$ \\
\hline
\end{tabular}




\begin{tabular}{|c|c|c|c|c|c|c|c|c|c|}
\hline & Pakistan & Peru & Russia & Singapore & South Korea & Taiwan & Thailand & The Philippines & United States \\
\hline $9^{\text {th }}$ group & $\begin{array}{c}1.40 \\
(1.648)\end{array}$ & $\begin{array}{c}4.10^{*} \\
(3.170)\end{array}$ & $\begin{array}{c}7.91 * * * \\
(6.218)\end{array}$ & $\begin{array}{c}11.83^{*} \\
(16.077)\end{array}$ & $\begin{array}{l}2.84 * * \\
(1.406)\end{array}$ & $\begin{array}{c}2.44 \\
(1.904)\end{array}$ & $\begin{array}{c}7.55^{* * *} \\
(3.117)\end{array}$ & $\begin{array}{c}1.74 \\
(0.744)\end{array}$ & $\begin{array}{c}3.79 * * * \\
(1.506)\end{array}$ \\
\hline Highest group & $\begin{array}{c}3.96 \\
(5.030)\end{array}$ & $\begin{array}{c}1.43 \\
(1.216)\end{array}$ & $\begin{array}{c}5.63 \\
(28.817)\end{array}$ & $\begin{array}{c}5.74 \\
(8.765)\end{array}$ & $\begin{array}{c}1.00 \\
(0.639)\end{array}$ & $\begin{array}{c}7.75 \\
(15.376)\end{array}$ & $\begin{array}{c}0.49 \\
(0.469)\end{array}$ & $\begin{array}{c}2.05 \\
(0.935)\end{array}$ & $\begin{array}{c}1.34 \\
(1.365)\end{array}$ \\
\hline Num of obs & 403 & 1048 & 1934 & 1837 & 1038 & 847 & 1107 & 1092 & 2097 \\
\hline Pseudo R2 & 0.036 & 0.0136 & 0.0265 & 0.011 & 0.0113 & 0.0127 & 0.0132 & 0.008 & 0.0090 \\
\hline Prob > chi2 & 0.000 & 0.000 & 0.000 & 0.000 & 0.000 & 0.000 & 0.000 & 0.007 & 0.000 \\
\hline
\end{tabular}

Source: Authors' calculation. *significant at $10 \%$ level, ** significant at $5 \%$ level, *** significant at $1 \%$ level. Robust standard error in parenthesis. To the education factor, the reference category is the incomplete primary school. The first level refers to complete primary school. The second level refers to an incomplete secondary school: technical/ vocational type. The third level refers to complete secondary school: technical/ vocational type. The fourth level refers to an incomplete secondary school: university-preparatory type. The fifth level refers to complete secondary school: university-preparatory type. The sixth level refers to some university-level education, without a degree and the seventh level refers to university - level education, with a degree 


\section{Table 3}

Ordered logit regression's result by countries. Coefficients are in forms of odds ratio.

\begin{tabular}{|c|c|c|c|c|c|c|c|c|c|c|}
\hline & Australia & Chile & China & Colombia & Hong Kong & India & Japan & Malaysia & Mexico & New Zealand \\
\hline Age & $\begin{array}{c}1.00 \\
(0.019)\end{array}$ & $\begin{array}{c}1.03 \\
(0.025)\end{array}$ & $\begin{array}{c}1.04 \\
(0.026)\end{array}$ & $\begin{array}{c}1.03 \\
(0.018)\end{array}$ & $\begin{array}{l}1.03 * \\
(0.021)\end{array}$ & $\begin{array}{c}1.02 \\
(0.021)\end{array}$ & $\begin{array}{c}1.02 \\
(0.020)\end{array}$ & $\begin{array}{c}0.98 \\
(0.021)\end{array}$ & $\begin{array}{c}1.00 \\
(0.016)\end{array}$ & $\begin{array}{c}0.98 \\
(0.030)\end{array}$ \\
\hline Age squared & $\begin{array}{c}1.00 \\
(0.000)\end{array}$ & $\begin{array}{c}1.00 \\
(0.000)\end{array}$ & $\begin{array}{c}1.00 * \\
(0.000)\end{array}$ & $\begin{array}{c}1.00 \\
(0.000)\end{array}$ & $\begin{array}{c}1.00 \\
(0.000)\end{array}$ & $\begin{array}{c}1.00 \\
(0.000)\end{array}$ & $\begin{array}{c}1.00 \\
(0.000)\end{array}$ & $\begin{array}{c}1.00 \\
(0.000)\end{array}$ & $\begin{array}{c}1.00 \\
(0.000)\end{array}$ & $\begin{array}{c}1.00 \\
(0.000)\end{array}$ \\
\hline Gender & $\begin{array}{c}1.19 \\
(0.141)\end{array}$ & $\begin{array}{c}1.00 \\
(0.136)\end{array}$ & $\begin{array}{c}1.03 \\
(0.111)\end{array}$ & $\begin{array}{c}1.31 * * * \\
(0.131)\end{array}$ & $\begin{array}{c}0.85 \\
(0.101)\end{array}$ & $\begin{array}{c}1.12 \\
(0.132)\end{array}$ & $\begin{array}{c}1.13 \\
(0.113)\end{array}$ & $\begin{array}{l}0.77 * * \\
(0.079)\end{array}$ & $\begin{array}{c}0.98 \\
(0.088)\end{array}$ & $\begin{array}{l}1.44 * * \\
(0.215)\end{array}$ \\
\hline Supervision & $\begin{array}{c}1.00 \\
(0.125)\end{array}$ & $\begin{array}{c}0.90 \\
(0.149)\end{array}$ & $\begin{array}{c}1.45 * * * \\
(0.195)\end{array}$ & $\begin{array}{c}1.10 \\
(0.122)\end{array}$ & $\begin{array}{c}0.94 \\
(0.122)\end{array}$ & $\begin{array}{c}0.68 * * * \\
(0.079)\end{array}$ & $\begin{array}{c}1.42 * * * \\
(0.139)\end{array}$ & $\begin{array}{c}1.46 * * * \\
(0.167)\end{array}$ & $\begin{array}{l}0.82 * * \\
(0.077)\end{array}$ & $\begin{array}{c}1.24 \\
(0.198)\end{array}$ \\
\hline \multicolumn{11}{|l|}{ Political Party } \\
\hline Inactive member & $\begin{array}{c}0.63 * * * \\
(0.113)\end{array}$ & $\begin{array}{c}0.91 \\
(0.180)\end{array}$ & $\begin{array}{c}1.19 \\
(0.265)\end{array}$ & $\begin{array}{c}1.02 \\
(0.190)\end{array}$ & $\begin{array}{c}1.07 \\
(0.190)\end{array}$ & $\begin{array}{c}1.04 \\
(0.126)\end{array}$ & $\begin{array}{c}0.84 \\
(0.171)\end{array}$ & $\begin{array}{c}0.99 \\
(0.137)\end{array}$ & $\begin{array}{l}1.34 * * \\
(0.189)\end{array}$ & $\begin{array}{l}0.61 * * \\
(0.142)\end{array}$ \\
\hline Active member & $\begin{array}{c}0.49 \\
(0.225)\end{array}$ & $\begin{array}{l}0.37 * * \\
(0.164)\end{array}$ & $\begin{array}{c}0.80 \\
(0.288)\end{array}$ & $\begin{array}{c}1.45 \\
(0.465)\end{array}$ & $\begin{array}{c}1.00 \\
(0.331)\end{array}$ & $\begin{array}{c}1.11 \\
(0.157)\end{array}$ & $\begin{array}{c}0.57 \\
(0.238)\end{array}$ & $\begin{array}{c}1.35 \\
(0.414)\end{array}$ & $\begin{array}{c}1.25 \\
(0.231)\end{array}$ & $\begin{array}{c}0.56 \\
(0.238)\end{array}$ \\
\hline \multicolumn{11}{|l|}{ Education } \\
\hline $1^{\text {st }}$ level & $\begin{array}{l}3.11 * \\
(2.077)\end{array}$ & $\begin{array}{l}2.57 * * \\
(0.964)\end{array}$ & - & $\begin{array}{c}1.30 \\
(0.268)\end{array}$ & $\begin{array}{c}0.81 \\
(0.406)\end{array}$ & $\begin{array}{c}1.35 \\
(0.257)\end{array}$ & - & $\begin{array}{c}0.92 \\
(0.296)\end{array}$ & $\begin{array}{c}0.99 \\
(0.224)\end{array}$ & - \\
\hline $2^{\text {nd }}$ level & $\begin{array}{c}2.36^{*} \\
(1.126)\end{array}$ & $\begin{array}{c}1.57 \\
(0.603)\end{array}$ & - & $\begin{array}{l}1.81 * * \\
(0.466)\end{array}$ & $\begin{array}{c}0.75 \\
(0.384)\end{array}$ & $\begin{array}{c}1.42 * \\
(0.291)\end{array}$ & $\begin{array}{c}1.55 \\
(0.804)\end{array}$ & $\begin{array}{c}1.29 \\
(0.426)\end{array}$ & $\begin{array}{c}1.12 \\
(0.326)\end{array}$ & - \\
\hline $3^{\text {rd }}$ level & $\begin{array}{c}2.26 * \\
(1.112)\end{array}$ & $\begin{array}{l}1.91 * * \\
(0.616)\end{array}$ & $\begin{array}{c}0.80 \\
(0.136)\end{array}$ & $\begin{array}{c}1.09 \\
(0.255)\end{array}$ & $\begin{array}{c}0.86 \\
(0.432)\end{array}$ & $\begin{array}{c}1.20 \\
(0.220)\end{array}$ & $\begin{array}{c}0.95 \\
(0.216)\end{array}$ & $\begin{array}{c}0.99 \\
(0.308)\end{array}$ & $\begin{array}{c}0.83 \\
(0.177)\end{array}$ & - \\
\hline $4^{\text {th }}$ level & $\begin{array}{c}1.63 \\
(0.901)\end{array}$ & $\begin{array}{c}1.34 \\
(0.634)\end{array}$ & - & $\begin{array}{c}1.27 \\
(0.267)\end{array}$ & $\begin{array}{c}0.88 \\
(0.549)\end{array}$ & $\begin{array}{c}0.93 \\
(0.208)\end{array}$ & $\begin{array}{c}0.77 \\
(0.216)\end{array}$ & $\begin{array}{c}1.64 \\
(0.698)\end{array}$ & $\begin{array}{c}1.01 \\
(0.248)\end{array}$ & $\begin{array}{c}4.31 \\
(6.448)\end{array}$ \\
\hline
\end{tabular}




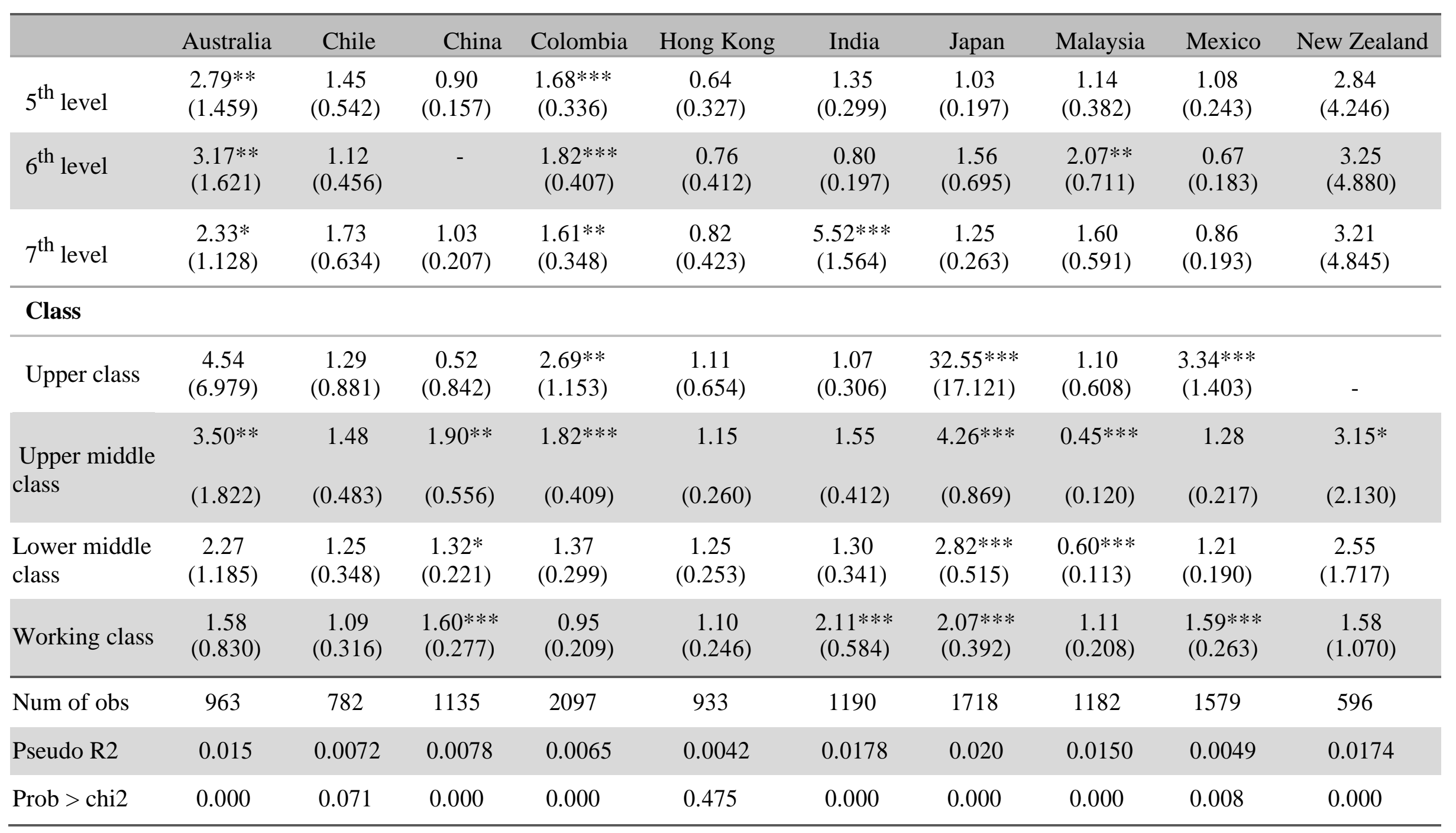




\section{Table 3}

Ordered logit regression's result by countries. Coefficients are in forms of odds ratio. (cont'd)

\begin{tabular}{|c|c|c|c|c|c|c|c|c|c|}
\hline & Pakistan - & Peru & $-\quad$ Russia & - Singapore & ${ }^{-}$South Korea & Taiwan & ${ }^{-}$Thailand ${ }^{-}$ & The Philippines & - United States \\
\hline Age & $\begin{array}{l}0.93 * * \\
(0.036)\end{array}$ & $\begin{array}{c}1.01 \\
(0.018)\end{array}$ & $\begin{array}{c}1.00 \\
(0.015)\end{array}$ & $\begin{array}{c}1.00 \\
(0.014)\end{array}$ & $\begin{array}{c}0.93 * * * \\
(0.022)\end{array}$ & $\begin{array}{c}0.98 \\
(0.023)\end{array}$ & $\begin{array}{c}1.05^{*} \\
(0.030)\end{array}$ & $\begin{array}{c}1.00 \\
(0.020)\end{array}$ & $\begin{array}{c}0.99 \\
(0.012)\end{array}$ \\
\hline Age squared & $\begin{array}{l}1.00 * * \\
(0.000)\end{array}$ & $\begin{array}{c}1.00 \\
(0.000)\end{array}$ & $\begin{array}{c}1.00 \\
(0.000)\end{array}$ & $\begin{array}{c}1.00 \\
(0.000)\end{array}$ & $\begin{array}{c}1.00 * * * \\
(0.000)\end{array}$ & $\begin{array}{c}1.00 \\
(0.000)\end{array}$ & $\begin{array}{c}1.00 * \\
(0.000)\end{array}$ & $\begin{array}{c}1.00 \\
(0.000)\end{array}$ & $\begin{array}{c}1.00 \\
(0.000)\end{array}$ \\
\hline Gender & $\begin{array}{c}0.44 \\
(0.321)\end{array}$ & $\begin{array}{c}1.01 \\
(0.114)\end{array}$ & $\begin{array}{l}1.09 * * \\
(0.092)\end{array}$ & $\begin{array}{c}0.98 \\
(0.083)\end{array}$ & $\begin{array}{c}1.08 \\
(0.121)\end{array}$ & $\begin{array}{c}0.97 \\
(0.129)\end{array}$ & $\begin{array}{c}1.01 \\
(0.109)\end{array}$ & $\begin{array}{c}0.96 \\
(0.109)\end{array}$ & $\begin{array}{l}1.24 * * * \\
(0.097)\end{array}$ \\
\hline Supervision & $\begin{array}{l}2.31 * * * \\
(0.499)\end{array}$ & $\begin{array}{c}1.14 \\
(0.155)\end{array}$ & $\begin{array}{c}1.27 \\
(0.136)\end{array}$ & $\begin{array}{c}1.30 * * * \\
(0.120)\end{array}$ & $\begin{array}{c}1.30 * \\
(0.180)\end{array}$ & $\begin{array}{c}1.06 \\
(0.148)\end{array}$ & $\begin{array}{c}0.93 \\
(0.120)\end{array}$ & $\begin{array}{c}1.20 \\
(0.155)\end{array}$ & $\begin{array}{c}1.14 \\
(0.093)\end{array}$ \\
\hline \multicolumn{10}{|l|}{ Political Party } \\
\hline $\begin{array}{l}\text { Inactive } \\
\text { member }\end{array}$ & $\begin{array}{l}2.59 * * \\
(1.066)\end{array}$ & $\begin{array}{c}0.76 \\
(0.188)\end{array}$ & $\begin{array}{c}1.40 \\
(0.397)\end{array}$ & $\begin{array}{c}0.86 \\
(0.120)\end{array}$ & $\begin{array}{c}0.73 \\
(0.179)\end{array}$ & $\begin{array}{c}1.23 \\
(0.196)\end{array}$ & $\begin{array}{c}0.88 \\
(0.193)\end{array}$ & $\begin{array}{c}1.12 \\
(0.202)\end{array}$ & $\begin{array}{c}1.03 \\
(0.090)\end{array}$ \\
\hline Active member & $\begin{array}{l}2.44 * * \\
(0.988)\end{array}$ & $\begin{array}{c}1.08 \\
(0.482)\end{array}$ & $\begin{array}{c}1.58 \\
(0.934)\end{array}$ & $\begin{array}{c}0.94 \\
(0.233)\end{array}$ & $\begin{array}{c}0.30 * \\
(0.214)\end{array}$ & $\begin{array}{c}1.37 \\
(0.492)\end{array}$ & $\begin{array}{l}1.81 * * \\
(0.541)\end{array}$ & $\begin{array}{c}1.29 \\
(0.297)\end{array}$ & $\begin{array}{c}1.06 \\
(0.136)\end{array}$ \\
\hline \multicolumn{10}{|l|}{ Education } \\
\hline $1^{\text {st }}$ level & $\begin{array}{c}2.32 * \\
(1.004)\end{array}$ & $\begin{array}{c}1.40 \\
(0.431)\end{array}$ & $\begin{array}{c}1.10 \\
(0.730)\end{array}$ & $\begin{array}{c}1.20 \\
(0.274)\end{array}$ & $\begin{array}{c}1.37 \\
(0.796)\end{array}$ & $\begin{array}{c}0.87 \\
(0.598)\end{array}$ & $\begin{array}{c}1.05 \\
(0.259)\end{array}$ & $\begin{array}{c}0.80 \\
(0.190)\end{array}$ & $\begin{array}{c}1.02 \\
(0.730)\end{array}$ \\
\hline $2^{\text {nd }}$ level & $\begin{array}{l}3.05 * * * \\
(1.309)\end{array}$ & $\begin{array}{c}1.45 \\
(0.406)\end{array}$ & $\begin{array}{c}1.58 \\
(0.931)\end{array}$ & $\begin{array}{c}0.99 \\
(0.251)\end{array}$ & $\begin{array}{c}1.68 \\
(1.325)\end{array}$ & $\begin{array}{l}0.19 * * \\
(0.145)\end{array}$ & $\begin{array}{c}1.40 \\
(0.588)\end{array}$ & $\begin{array}{c}0.79 \\
(0.232)\end{array}$ & - \\
\hline $3^{\text {rd }}$ level & $\begin{array}{l}2.79 * * \\
(1.207)\end{array}$ & $\begin{array}{c}2.00 * * * \\
(0.511)\end{array}$ & $\begin{array}{c}0.90 \\
(0.493)\end{array}$ & $\begin{array}{c}1.22 \\
(0.247)\end{array}$ & $\begin{array}{c}0.91 \\
(0.470)\end{array}$ & $\begin{array}{c}0.64 \\
(0.445)\end{array}$ & $\begin{array}{c}1.27 \\
(0.360)\end{array}$ & $\begin{array}{c}0.70 \\
(0.174)\end{array}$ & - \\
\hline $4^{\text {th }}$ level & $\begin{array}{c}3.35^{*} \\
(2.391)\end{array}$ & $\begin{array}{l}2.15 * * \\
(0.681)\end{array}$ & $\begin{array}{c}1.06 \\
(0.592)\end{array}$ & $\begin{array}{c}1.25 \\
(0.304)\end{array}$ & $\begin{array}{c}1.15 \\
(0.652)\end{array}$ & $\begin{array}{c}0.53 \\
(0.460)\end{array}$ & $\begin{array}{c}0.81 \\
(0.374)\end{array}$ & $\begin{array}{c}1.14 \\
(0.317)\end{array}$ & $\begin{array}{c}0.72 \\
(0.439)\end{array}$ \\
\hline $5^{\text {th }}$ level & $\begin{array}{l}3.13 * * \\
(1.565)\end{array}$ & $\begin{array}{c}2.59 * * * \\
(0.751)\end{array}$ & $\begin{array}{c}0.75 \\
(0.413)\end{array}$ & $\begin{array}{c}0.93 \\
(0.207)\end{array}$ & $\begin{array}{c}1.22 \\
(0.605)\end{array}$ & $\begin{array}{c}0.61 \\
(0.420)\end{array}$ & $\begin{array}{c}0.82 \\
(0.265)\end{array}$ & $\begin{array}{c}1.00 \\
(0.238)\end{array}$ & $\begin{array}{c}0.71 \\
(0.425)\end{array}$ \\
\hline
\end{tabular}




\begin{tabular}{|c|c|c|c|c|c|c|c|c|c|}
\hline $6^{\text {th }}$ level & $\begin{array}{c}3.38 \\
(2.571)\end{array}$ & $\begin{array}{c}2.69 * * * \\
(0.812)\end{array}$ & $\begin{array}{c}1.15 \\
(0.666)\end{array}$ & $\begin{array}{c}0.72 \\
(0.196)\end{array}$ & $\begin{array}{c}1.20 \\
(0.626)\end{array}$ & $\begin{array}{c}1.12 \\
(0.886)\end{array}$ & $\begin{array}{c}0.36 \\
(0.359)\end{array}$ & $\begin{array}{c}0.95 \\
(0.214)\end{array}$ & $\begin{array}{c}0.69 \\
(0.412)\end{array}$ \\
\hline $7^{\text {th }}$ level & $\begin{array}{l}5.01 * * * \\
(2.696)\end{array}$ & $\begin{array}{c}2.40 * * * \\
(0.675)\end{array}$ & $\begin{array}{c}0.97 \\
(0.538)\end{array}$ & $\begin{array}{c}1.00 \\
(0.219)\end{array}$ & $\begin{array}{c}0.94 \\
(0.474)\end{array}$ & $\begin{array}{c}0.64 \\
(0.444)\end{array}$ & $\begin{array}{c}0.65 \\
(0.186)\end{array}$ & $\begin{array}{c}1.40 \\
(0.333)\end{array}$ & $\begin{array}{c}0.54 \\
(0.321)\end{array}$ \\
\hline \multicolumn{10}{|l|}{ Class } \\
\hline Upper class & $\begin{array}{c}0.80 \\
(0.526)\end{array}$ & $\begin{array}{c}0.68 \\
(0.409)\end{array}$ & $\begin{array}{l}6.10 * * * \\
(3.434)\end{array}$ & $\begin{array}{c}2.59 * * * \\
(0.835)\end{array}$ & $\begin{array}{c}2.31 \\
(1.386)\end{array}$ & $\begin{array}{l}6.83 * * \\
(6.693)\end{array}$ & $\begin{array}{c}0.57 \\
(0.848)\end{array}$ & $\begin{array}{c}0.85 \\
(0.280)\end{array}$ & $\begin{array}{l}2.69 * * \\
(1.153)\end{array}$ \\
\hline $\begin{array}{l}\text { Upper middle } \\
\text { class }\end{array}$ & $\begin{array}{c}0.76 \\
(0.257)\end{array}$ & $\begin{array}{c}0.94 \\
(0.194)\end{array}$ & $\begin{array}{c}2.97 * * * \\
(0.595)\end{array}$ & $\begin{array}{l}3.28 * * * \\
(0.847)\end{array}$ & $\begin{array}{l}2.35 * * \\
(0.870)\end{array}$ & $\begin{array}{l}3.15 * * \\
(1.651)\end{array}$ & $\begin{array}{c}1.57 * \\
(0.408)\end{array}$ & $\begin{array}{c}0.99 \\
(0.194)\end{array}$ & $\begin{array}{l}1.82 * * * \\
(0.409)\end{array}$ \\
\hline $\begin{array}{l}\text { Lower middle } \\
\text { class }\end{array}$ & $\begin{array}{c}1.37 \\
(0.436)\end{array}$ & $\begin{array}{c}0.93 \\
(0.170)\end{array}$ & $\begin{array}{c}2.04 * * * \\
(0.345)\end{array}$ & $\begin{array}{c}2.80 * * * \\
(0.693)\end{array}$ & $\begin{array}{c}1.36 \\
(0.493)\end{array}$ & $\begin{array}{c}1.54 \\
(0.786)\end{array}$ & $\begin{array}{c}0.89 \\
(0.216)\end{array}$ & $\begin{array}{l}0.66 * * \\
(0.117)\end{array}$ & $\begin{array}{c}1.37 \\
(0.299)\end{array}$ \\
\hline Working class & $\begin{array}{c}3.23 * * * \\
(1.226)\end{array}$ & $\begin{array}{c}0.99 \\
(0.180)\end{array}$ & $\begin{array}{c}2.27 * * * \\
(0.381)\end{array}$ & $\begin{array}{c}2.15 * * * \\
(0.558)\end{array}$ & $\begin{array}{c}1.68 \\
(0.637)\end{array}$ & $\begin{array}{c}1.44 \\
(0.724)\end{array}$ & $\begin{array}{c}0.99 \\
(0.248)\end{array}$ & $\begin{array}{c}0.92 \\
(0.199)\end{array}$ & $\begin{array}{c}0.95 \\
(0.209)\end{array}$ \\
\hline Num of obs & 403 & 1048 & 1934 & 1837 & 1038 & 847 & 1107 & 1092 & 2097 \\
\hline Pseudo R2 & 0.042 & 0.0059 & 0.0272 & 0.0075 & 0.0104 & 0.0130 & 0.009 & 0.0079 & 0.0065 \\
\hline Prob > chi2 & 0.000 & 0.040 & 0.000 & 0.000 & 0.000 & 0.000 & 0.001 & 0.002 & 0.000 \\
\hline
\end{tabular}

Source: Authors' calculation. *significant at 10\% level, $* *$ significant at $5 \%$ level, $* * *$ significant at $1 \%$ level. Robust standard error in parenthesis. To the education factor, the reference category is the incomplete primary school. The first level refers to complete primary school. The second level refers to an incomplete secondary school: technical/ vocational type. The third level refers to complete secondary school: technical/ vocational type. The fourth level refers to an incomplete secondary school: university-preparatory type. The fifth level refers to complete secondary school: university-preparatory type. The sixth level refers to some university-level education, without degree and the seventh level refers to university - level education, with a degree. To the class factor, the reference category is the lower class 


\section{Concluding remarks and policy implications}

This empirical study aims to determine drivers of attitude toward income inequality from a pool of demographic factors across the selected countries in the Asia-Pacific region, using the most recent data from World Values Survey in 2016. Various demographic factors, including Gender, Age, Political party, Education, Supervision, Family income, and Class are included in this study. The findings from this empirical study suggest that the role of each demographic factor as a significant explanation of variation in the attitude toward income inequality depends on the selected countries. In other words, the impact of demographic factors on attitude toward income inequality varies by countries. In addition, the components of a set of demographic factors, significantly contributing to the variation in selected countries in the study. Remarkably, among the demographic factors, as family income is employed, Supervision is a significant contribution to attitude toward income inequality in 9 countries, and as Class is taken into account, the role of Supervision is found statistically significant in 8 countries.

On the ground of key findings from this study, social and economic policies targeted to the attitude toward income inequality in Vietnam in the future will need to focus on the socalled Class such as Upper Class; Working Class; or Lower Class in the society. Doing so will reduce the gaps between classes or even to eliminate them in order to achieve a more harmonic society in the process of economic growth and development in Vietnam in the near future.

\section{References}

Austen, S. (2002). An international comparison of attitudes to inequality. International Journal of Social Economics, 29(3), 218-237. doi:10.1108/03068290210417106

Cingano, F. (2014). Trends in income inequality and its impact on economic growth (OECD Social, Employment and Migration Working Papers, No. 163). doi:10.1787/5jxrjncwxv6j-en

Curtis, J., \& Andersen, R. (2015). How social class shapes attitudes on economic inequality: The competing forces of self-interest and legitimation. International Review of Social Research, 5(1), 4-19. doi:10.1515/irsr-2015-0002

Cyba, E. (2000). Geschlecht und soziale Ungleichheit. Konstellationen der Frauenbenachteiligung. Opladen, Germany: Leske und Budrich.

Dabla-Norris, E., Tsounta, E., Kochhar, K., Ricka, F., \& Suphaphiphat, N. (2015). Causes and consequences of income inequality: A global perspective. Washington, DC: International Monetary Fund.

Dorling, D. (2011). Injustice: Why social inequality persists. Bristol, UK: Policy Press.

Frerichs, P. (1997). Klasse und Geschlecht 1. Arbeit. Macht. Anerkennung. Interessen. Opladen, Germany: Leske und Budrich.

Gijsberts, M. (2002). The legitimation of income inequality in state-socialist and market societies. Acta Sociologica, 45(4), 269-285. doi:10.1177/000169930204500402 
Hirschman, A. O., \& Rothschild, M. (1973). The changing tolerance for income inequality in the course of economic development. The Quarterly Journal of Economics, 87(4), 544566. doi: $10.2307 / 1882024$

Kelley, J., \& Evans, M. D. (1993). The legitimation of inequality: Occupational earnings in nine nations. American Journal of Sociology, 99(1), 75-125.

Kim, H., Huh, S., Choi, S., \& Lee, Y. (2018). Perceptions of inequality and attitudes towards redistribution in four East Asian welfare states. International Journal of Social Welfare, 27(1), 28-39. doi:10.1111/ijsw.12266

Mau, S. (1997). Ideologischer konsens und dissens im wohlfahrtsstaat: Zur binnenvariation von einstellungen $\mathrm{zu}$ sozialer ungleichheit in Schweden, Großbritannien und der Bundesrepublik Deutschland. Soziale Welt, 48(1), 17-37.

Medgyesi, M. (2013). Increasing income inequality and attitudes to inequality: A cohort perspective (AIAS, GINI Discision Paper, No. 94). Retrieved March 10, 2018, from https://www.researchgate.net/publication/312266723_Increasing_income_inequality_a nd_attitudes_to_inequality_a_cohort_perspective

Noll, H. H. (1998). Wahrnehmung und Rechtfertigung sozialer Ungleichheit 1991-1996. Opladen, Germany: Leske und Budrich.

Stiglitz, J. (2012). The Price of Inequality: How today's divided society endangers our future. New York, NY: W. W. Norton \& Company. 